\title{
Produtividade do tomateiro em cultivo solteiro e consorciado com espécies aromáticas e medicinais
}

\author{
Luciana M de Carvalho'; Maria Urbana C Nunes; Ivênio Rubens de Oliveira; Maria de Lourdes da S \\ Leal \\ Embrapa Tabuleiros Costeiros, C. Postal 44, 49025-480 Aracaju-SE; ' ${ }^{1}$ uttor correspondente; luciana@cpatc.embrapa.br; murbana@cpatc. \\ embrapa.br;ivenio@cpatc.embrapa.br; lurdinha@cpatc.embrapa.br
}

\begin{abstract}
RESUMO
O tomateiro é cultivado, principalmente em monocultivos, com uso intensivo de defensivos químicos, atividade de risco econômico, social e ambiental. O consórcio vem sendo praticado como forma de reduzir os riscos. O objetivo deste trabalho foi avaliar a produção do tomateiro solteiro e em consórcio com as espécies medicinais e aromáticas Foeniculum vulgare, Mentha piperita, Ocimum basilicum e Ruta graveolens. O delineamento experimental foi de blocos ao acaso com quatro repetições e cinco tratamentos. Avaliou-se altura, área do dossel, produção, produtividade e eficiência do uso da terra. Nos consórcios, verificou-se que, na densidade de plantio adotada, o funcho teve maior altura e área de dossel e causou redução significativa na produção total de tomates: de $12,23 \mathrm{~kg}$, no cultivo solteiro, para $7,88 \mathrm{~kg}$, no consórcio com funcho. No entanto, contribuiu com a menor porcentagem de perda de tomates por broqueamento (24\%). As maiores perdas por broqueamento ocorreram no monocultivo e no consórcio com hortelã-pimenta. A arruda favoreceu aumento significativo na produção comercial de tomates, em média $26 \%$. A produtividade relativa do tomateiro foi maior no consórcio com arruda (13,6 tha $\left.\mathrm{th}^{-1}\right)$, seguido pelo consórcio com hortelã-pimenta $(9,8$ $\mathrm{t}$ ha $\left.{ }^{-1}\right)$ e manjericão $\left(9,1 \mathrm{t} \mathrm{ha}^{-1}\right)$, e menor no consórcio com funcho $\left(6,4 \mathrm{t} \mathrm{ha}^{-1}\right)$, o que indicou que o consórcio com arruda foi mais vantajoso para a produção de tomates. Dentre as aromáticas estudadas, o manjericão teve maior produtividade $\left(96,5 \mathrm{t} \mathrm{ha}^{-1}\right)$. Concluindo, os consórcios permitiram maior aproveitamento no uso da terra, com aumento na produtividade dos tomateiros na presença da arruda e redução na presença do funcho. As demais plantas não reduziram significativamente a produção e portanto podem ser empregadas como fonte alternativa de renda.
\end{abstract}

Palavras-chave: Lycopersicum esculentum, Foeniculum vulgare, Mentha piperita, Ocimum basilicum, Ruta graveolens, consorciação de culturas, competição.

\section{ABSTRACT \\ Yield of tomato in monocrop and intercropping with aromatics plants}

Tomato is mainly cultivated in monocrops, by intensive use of chemical products. It is an activity with economic, social and environmental risks. The intercropping has been used as a form to reduce these risks. In this work the yield of tomato in sole crop and intercropping with Foeniculum vulgare, Mentha piperita, Ocimum basilicum and Ruta graveolens was evaluated. The monocrop of tomato and its intercropping was evaluated with aromatic plants in a complete randomized design, with five treatments and four blocks. We evaluated height, canopy area, production, yield and land equivalent ratio. On the population density adopted, the $F$. vulgare (fennel) presented greater height and canopy area, and caused significant reduction on total production of tomato, from $12.23 \mathrm{~kg}$, in monocrop, to $7.88 \mathrm{~kg}$, when intercropped with fennel. However, intercropping with fennel contributed to a lesser loose caused by fruits borer $(24 \%)$. The greatest loose caused by fruits borer occurred in monocropping and in intercropping with $M$. piperita (peppermint). Intercropping with Ruta graveolens (rue) resulted significant increase of commercial production of fruits, about $26 \%$. The relative yield of tomato was greater in intercropping with rue $\left(13.6 \mathrm{t} \mathrm{ha}^{-1}\right)$, followed by intercropping with peppermint $\left(9.8 \mathrm{tha}^{-1}\right)$ and with $O$. basilicum (basil) (9.1 t ha-1), and lesser on intercropping with fennel (6.4 t $\left.\mathrm{ha}^{-1}\right)$. These results showed that the intercropping with rue was more advantageous than others producing more fruits. Among the studied aromatic plants, basil presented the highest yield $\left(96.5 \mathrm{t} \mathrm{ha}^{-1}\right)$. The tomato intercropping with aromatic cultures permits better use of land and higher yield, mainly when intercropping with rue. All aromatics plants evaluated represent an alternative source of gain.

Keywords: Lycopersicum esculentum, Foeniculum vulgare, Mentha piperita, Ocimum basilicum, Ruta graveolens, intercropping, competition.

(Recebido para publicação em 23 de setembro de 2008; aceito em 17 de julho de 2009)

(Received in September 23, 2008; accepted in July 17, 2009)

\begin{abstract}
$\mathrm{A}^{\mathrm{i}}$ intensificação da agricultura tem contribuído, substancialmente, no aumento da produção de alimentos nos últimos 50 anos. Entretanto, os efeitos ecológicos colaterais podem ser tão profundos quanto o aumento da produção de alimentos (Matson et al., 1997). Nas últimas décadas, o nível de conscientização das relações da agricultura
\end{abstract}

com o ambiente, os recursos naturais e a qualidade dos alimentos cresceu e tem sido demandada mudança para uma forma de produção de alimentos mais sustentável (Hiddink et al., 2005; Oliveira et al., 2005).

Dentre as práticas alternativas de produção enquadram-se os consórcios, que são definidos como sistemas de cul- tivo em que há o crescimento simultâneo de duas ou mais espécies de plantas na mesma área, com o fim de permitir interação biológica benéfica entre elas (Vandermeer, 1989). Podem resultar em aumento da produtividade, da eficiência de uso dos recursos disponíveis, da estabilidade econômica e biológica do agroecossistema, e na redução da infes- 
tação com plantas invasoras e da pressão de pragas e doenças (Vandermeer, 1989; Jolliffe \& Wanjau, 1999; Altieri et al., 2003; Hiddink et al., 2005).

A consorciação de culturas é bastante comum entre pequenos produtores, especialmente nos países tropicais (Altieri et al., 2003; Sobkowicz \& Tendziagolska, 2005).

Associações entre hortaliças, apesar de comuns, têm sido ainda pouco estudadas (Santos et al., 2002; Oliveira et al., 2005; Teixeira et al., 2005; Montezano \& Peil, 2006; Moraes et al., 2007; Grangeiro et al., 2008). Atualmente, os maiores desafios da pesquisa se relacionam à determinação das culturas a serem cultivadas juntas, à forma de manejo e à viabilidade destes sistemas (Montezano \& Peil, 2006).

Nos últimos anos surgiram alguns estudos de consorciação de hortaliças com espécies medicinais (Rao, 1999; Patra et al., 2002; Gómez-Rodríguez et al., 2003; Bomford, 2004; Moraes et al., 2007; Nascimento et al., 2007). Paralelamente, surgiu uma corrente de estudiosos dedicados à busca das propriedades inseticida, repelente ou iscas atrativas para insetos entre as espécies aromáticas (Bowie et al., 1995; Roel, 2001; Rao, 2002). Mas ainda são poucos os estudos de consórcio de hortaliças com aromáticas (Maia et al., 2008). Investigações científicas comparando consórcios do tomateiro com diferentes espécies aromáticas não foram encontrados.

O tomateiro (Lycopersicum esculentum Mill.) é a hortaliça mais cultivada no Brasil (Souza \& Resende, 2003). Nas regiões tropicais, a produção é influenciada por expressivas reduções de rendimento e depreciação da qualidade dos frutos, em razão da ocorrência de doenças, pragas e estresses abióticos (Melo \& Vilela, 2005).

A alta suscetibilidade ao ataque de pragas e a exigência em insumos e serviços elevam a necessidade de investimento financeiro, o que torna o cultivo atividade agrícola de alto risco (Loos et al., 2004).

As espécies Foeniculum vulgare (funcho), Mentha piperita (hortelãpimenta), Ocimum basilicum (manjeri- cão) e Ruta graveolens (arruda) são de múltiplo uso: medicinais, aromáticas, condimentares, repelentes, companheiras, entre outros.

O manjericão é relatado como espécie "companheira" do tomateiro, favorecendo a produção de frutos (Bomford, 2004). O funcho, mais conhecido na região Nordeste como erva-doce, é cultivado em alguns estados do Nordeste como fonte de renda alternativa na agricultura familiar, sendo citado como espécie "antagonista" do tomateiro, ou seja, que prejudica seu crescimento e desenvolvimento (Gamarra-Rojas, 2003). Arruda e hortelã-pimenta são plantas comumente utilizadas em preparações caseiras para controle natural de pragas, além de terem utilidade e demanda como medicinal (Burg \& Mayer, 2006).

A medida mais utilizada para avaliar a eficiência de um consórcio é a razão de equivalência de áreas ou produtividade relativa total (Vandermeer, 1989). Altieri et al. (2003) definem esse índice como área relativa requerida para policulturas produzirem os mesmos rendimentos das culturas solteiras.

Em função da tradução esse índice tem recebido diferentes nomes e abreviaturas: Índice de equivalência de áreas, IEA (Gliessman, 2001; Santos et al., 2002), Taxa de uso eficiente da terra (Altieri et al., 2003) e Índice de uso eficiente da terra, UET (Azevedo et al., 1998).

Atualmente, as preocupações com o ambiente e a qualidade de vida têm difundido amplamente as correntes de agricultura alternativa, dentre elas, destaca-se a agricultura orgânica. Esse sistema de produção tem crescido continuamente, em função de demanda cada vez maior.

O Brasil ocupa a $13^{a}$ posição mundial quanto à área destinada à agricultura orgânica certificada, com mais de 275 mil hectares. Dentre os alimentos produzidos, destacam-se as hortaliças para o mercado interno.

Diante da necessidade de incentivar o cultivo orgânico e da necessidade de determinar estratégias tecnológicas para colaborar na redução de riscos e perdas na produtividade, este trabalho teve o objetivo de estudar o efeito do consórcio do tomateiro com plantas aromáticas sobre o crescimento e produtividade destas espécies.

\section{MATERIAL E MÉTODOS}

O experimento foi conduzido no município de Umbaúba-SE (11 22 '37"S; 3740’26" O; $109 \mathrm{~m}$ de altitude), de março, quando se iniciou a produção de mudas, a dezembro de 2006, com o término das colheitas. O clima local é caracterizado, segundo a classificação de Köppen, como AS tropical chuvoso com verão seco.

O solo é classificado como Argissolo acinzentado Eutrófico com fragipan $\mathrm{Tb}$ A fraco, textura média/argilosa. Os resultados da análise química do solo foram; $13,9 \mathrm{~g} \mathrm{dm}^{-3}$ de matéria orgânica, $4,70 \mathrm{ppm}$ de fósforo, $0,17,0,92,1,56$, $0,05 \mathrm{cmolc} \mathrm{dm}^{-3}$, respectivamente, de potássio, magnésio, cálcio e alumínio, pH em água de 6,4, soma de bases trocáveis de $2,68 \mathrm{cmolc} \mathrm{dm}^{-3}$ e índice de saturação de bases de $65,2 \%$.

Foram estudadas quatro plantas aromáticas (arruda, funcho, hortelã-pimenta e manjericão), em cultivo solteiro e consorciado com o tomateiro, e o cultivo solteiro de tomateiro. O experimento foi instalado no delineamento experimental de blocos ao acaso, com quatro repetições e cinco tratamentos.

Os tratamentos incluíram o cultivo solteiro do tomateiro e os consórcios do tomateiro com arruda, hortelã- pimenta, funcho e manjericão. Adicionalmente, foram instalados os tratamentos das aromáticas em cultivo solteiro, a fim de possibilitar o cálculo do UET.

A cultivar de tomateiro utilizada foi Caline IPA-6, industrial melhorada para mesa, desenvolvida pela Empresa Pernambucana de Pesquisa Agropecuária (IPA). Apesar de ser variedade rasteira é utilizada como tomate de mesa na região. Foi conduzida semi-tutorada, por meio do amarrio em estaca de madeira vertical, prática adotada pelos agricultores da região.

Com o fim de reduzir a ação do vento, de aumentar a biodiversidade local e contribuir com o controle de pragas, implantou-se barreiras vivas no entorno de cada parcela e da área experimental como um todo. No entorno das parcelas 
foi instalado cordão de feijão guanduanão (Cajanus cajan), mantido com 1,2 $\mathrm{m}$ de altura por meio de podas periódicas; e, no entorno da área experimental, à distância de 4,5 $\mathrm{m}$ das parcelas foi instalada uma barreira viva constituída de linha de maracujá (Passiflora $\mathrm{sp}$ ), de mamoeiro (Carica papaya) e de crotalaria (Crotalaria spectabilis).

A área total da parcela foi $16,2 \mathrm{~m}^{2}$ (5,4 $\mathrm{m}$ de largura $\mathrm{x} 3 \mathrm{~m}$ de comprimento), sendo considerada nas avaliações dos tomateiros a área útil de $6 \mathrm{~m}^{2}$, espaço ocupado por dez plantas de tomateiro, arruda, manjericão e funcho e 20 de hortelã, distribuídas nas linhas centrais da parcela.

Dentro das linhas de plantio, os espaçamentos entre plantas foram de 0,6 $\mathrm{m}$, para o tomateiro, arruda e manjericão e de $0,3 \mathrm{~m}$, para hortelã-pimenta. O consórcio com aromáticas foi estabelecido aproveitando-se o espaço entre duas linhas de plantio de tomateiro para acrescentar uma linha de aromática, no esquema de consórcio aditivo descrito por Vandermeer (1989). Assim, o espaçamento entre linhas foi de $1,0 \mathrm{~m}$ e 0,5 $\mathrm{m}$, respectivamente, nas parcelas de monocultivo e de consórcio.

As sementes foram adquiridas no comércio local e as mudas de todas as espécies foram produzidas, em ambiente protegido, utilizando bandejas de poliestireno, preenchidas com substrato natural à base de pó de casca de coco, esterco e pó de rocha.

A análise química do substrato foi 6,4 de $\mathrm{pH}$ em água, $102 \mathrm{~g} \mathrm{dm}^{-3}$ de matéria orgânica; 23,2; 9,30;0,05;0,835; 3,54 cmolc $\mathrm{dm}^{-3}$ de cálcio, magnésio, alumínio, sódio e potássio respectivamente; $6.350 \mathrm{ppm}$ de fósforo; 36,9 cmolc $\mathrm{dm}^{-3}$ de soma de bases trocáveis e $96,8 \%$ de índice de saturação de bases. Como as aromáticas estudadas têm um desenvolvimento mais lento do que o tomateiro, estas foram semeadas em março e transplantadas no final de abril, 20 dias antes do transplantio do tomateiro.

O início do experimento foi definido com o transplantio das mudas de tomateiro e o estabelecimento dos consórcios, em maio. O trasplantio do tomateiro e do manjericão ocorreu 25 dias após a semeadura, enquanto o do funcho, hortelã-pimenta e arruda foi aos 40 dias após semeadura.

O preparo do solo foi feito com aração e gradagem leves. A adubação de plantio foi feita, com base nas necessidades das culturas em estudo e em análise de solo, com hiperfosfato de gafsa $(\mathrm{P}$, $2730 \mathrm{ppm}$ ), torta de mamona (K, 12,8 cmolc $\mathrm{dm}^{-3}$ ), sulfato de potássio, gesso agrícola e cinza produzida em casa de farinha, nas doses correspondentes a $100 \mathrm{~kg} \mathrm{ha}^{-1} \mathrm{~N}, 400 \mathrm{~kg} \mathrm{ha}^{-1} \mathrm{P}_{2} \mathrm{O}_{5}, 150 \mathrm{~kg}$ $\mathrm{ha}^{-1}$ e $1,0 \mathrm{t} \mathrm{ha}^{-1}$ de gesso agrícola, além de $20 \mathrm{t} \mathrm{ha}^{-1}$ de esterco bovino curtido, e incorporadas nos camalhões de plantio (EMBRAPA, 1987; Sobral et al., 2007). Em cobertura, aos 30 e 60 dias após transplantio, foram adicionados $40 \mathrm{~kg}$ $\mathrm{ha}^{-1} \mathrm{~N}$, utilizando torta de mamona e 10 $\mathrm{t} \mathrm{ha}^{-1}$ de esterco bovino curtido a todas as culturas.

Os tomateiros receberam ainda, em cobertura, sulfato de potássio na dose de $50 \mathrm{~kg} \mathrm{ha}^{-1}$ de $\mathrm{K}_{2} \mathrm{O}$. A irrigação foi por gotejamento, com fitas gotejadoras instaladas junto às linhas de plantio, com gotejadores distanciados em 0,3 m (vazão de 1,4 $\mathrm{L} \mathrm{h}^{-1}$ ).

A altura e área do dossel das plantas de tomateiro, arruda, funcho, hortelãpimenta e manjericão foram avaliadas quando se detectou início de frutificação em $50 \%$ dos tomateiros e surgiram os primeiros frutos maduros, aos 56 dias após transplantio dos tomateiros.

A área do dossel de cada planta foi estimada, segundo Santos et al. (2002), pelo produto das duas maiores dimensões do dossel, tomadas ao longo da linha de plantio e no seu ângulo reto. Além disso, durante todo o experimento, foi feita avaliação da entomofauna associada, em todas as parcelas experimentais.

A fim de não prejudicar a observação de possíveis efeitos das aromáticas na redução de perdas na produção, não foi utilizado nenhum produto para controle de pragas ou doenças.

Foram realizadas seis colheitas de tomates no período de 31 de outubro, aos 56 dias após o transplantio, a 5 de dezembro de 2006. Foram colhidos, semanalmente, desde frutos em início de maturação (com mudança de coloração de verde para amarelo) até frutos ma- duros (casca completamente vermelha). Frutos danificados, incluindo aqueles com rachaduras, com podridão apical (deficiência de cálcio) ou com danos causados por insetos foram considerados não-comerciais.

Foi registrada a massa fresca dos frutos comerciais e não-comerciais, dos frutos danificados por broca pequena ou grande e dos frutos com sintomas de deficiência de cálcio, segundo metodologia de Picanço et al. (1997) e Loos et al. (2004). Foram estimadas as produtividades absoluta e relativa, segundo Vandermeer (1989).

O manjericão foi a primeira aromática a ser colhida, seguido pela arruda, hortelã-pimenta e funcho. A colheita de manjericão, arruda e hortelã foi feita em $50 \%$ dos ramos, com auxílio de tesoura de poda, sendo colhidos ramos com cerca de 15 a $20 \mathrm{~cm}$ de comprimento. No caso do funcho todos os frutos verde-amarelados das plantas úteis foram colhidos. A época de colheita foi definida segundo Martins et al. (1995). Para efeito das avaliações de produtividade realizadas foram consideradas as colheitas de frutos do funcho (realizadas em dezembro), de ramos foliares de manjericão (realizadas mensalmente de agosto a dezembro), de arruda (em novembro) e de hortelã-pimenta (em dezembro). Similarmente ao procedimento adotado para o tomateiro, determinou-se a produção de massa fresca das aromáticas, a produtividade absoluta e a produtividade relativa.

Com a soma das produtividades relativas do tomateiro e da aromática consorciada, obtidos na mesma área, foi calculado o UET, segundo metodologia descrita por Vandermeer (1989). Os dados foram submetidos à análise de variância e teste Tukey, por meio do programa estatístico SAS.

Dados de porcentagem de perda de produção de tomates devido ao broqueamento, antes de serem submetidos à análise estatística, foram transformados em arco seno, a fim de ajustar a homogeneidade e normalidade. Os dados de UET foram segundo orientação proposta em Vandermeer (1989) corrigidos pela média da produtividade absoluta dos cultivos solteiros.

Em dezembro, após término das 
colheitas de tomate e arranquio das plantas, foi semeado nas mesmas linhas de plantio, feijão de porco (Canavalia ensiformes) e girassol (Helianthus annuus), como adubo verde. Em janeiro e fevereiro foram feitas as últimas colheitas de manjericão, em fevereiro as últimas de arruda, e em março as últimas de hortelã-pimenta. Nas avaliações de produção das aromáticas, no entanto, só foram consideradas as colheitas obtidas até dezembro, quando se colheram os tomates.

\section{RESULTADOS E DISCUSSÃO}

Aos 56 dias após implantação dos consórcios verificou-se que apenas a altura das plantas de funcho foi influenciada positivamente pelo consórcio: 112,28 $\mathrm{cm}$ nas plantas em cultivo consorciado e 89,28 cm nas plantas em monocultivo (Tabela 1). Supõe-se que essas plantas têm nichos ecológicos similares ao do tomateiro, o que favoreceu o aumento da pressão competitiva e o maior crescimento em altura, como estratégia de defesa.

Segundo Vandermeer (1989), quanto mais similares os requerimentos de duas espécies, definidos pelo nicho ecológico, maior a competição entre elas. A partir desse resultado e considerando que o espaçamento entre linhas de plantio foi de apenas $0,5 \mathrm{~m}$, sugere-se que houve sombreamento de plantas, no consórcio do tomateiro com o funcho.

A frutificação dos tomateiros foi detectada, primeiramente, nas plantas consorciadas com o funcho, aos 28 dias após transplantio. Aos 56 dias, cerca de $50 \%$ dos tomateiros tinham iniciado a frutificação e já havia alguns poucos frutos maduros.

A produção total de tomates foi significativamente menor nos tomateiros consorciados com funcho, que não diferiu significativamente, no entanto, dos valores obtidos nos tomateiros solteiros ou consorciados com hortelã-pimenta e manjericão (Tabela 2). Sugere-se que a menor produção ocorreu, ao menos em parte, devido aos efeitos do sombreamento e da competição das duas espécies por luz e nutrientes. Analisando-se a produção total de tomates da parcela útil, constataram-se valores significativamente superiores nos tomateiros

Tabela 1. Altura e área do dossel de plantas de arruda, funcho, hortelã-pimenta e manjericão, em cultivo solteiro e consorciado, 56 dias após instalação dos consórcios (height and dossel area of rue, fennel, peppermint and basil, in single and intercropping, at 56 days after setting the experiment). Umbaúba, Embrapa Tabuleiros Costeiros, 2006.

\begin{tabular}{lcclrr}
\hline \multirow{2}{*}{ Cultura } & \multicolumn{2}{c}{ Altura $(\mathbf{c m})$} & & \multicolumn{2}{c}{ Área do dossel $\left.\mathbf{( c m}^{2}\right)$} \\
\cline { 2 - 3 } \cline { 5 - 6 } & Consórcio & Solteiro & & Consórcio & Solteiro \\
\hline Arruda & $36,1 \mathrm{~A}$ & $30,3 \mathrm{~A}$ & & $965,5 \mathrm{~A}$ & $1038,1 \mathrm{~A}$ \\
Funcho & $112,3 \mathrm{~A}$ & $89,3 \mathrm{~B}$ & & $4842,7 \mathrm{~A}$ & $4873,3 \mathrm{~A}$ \\
Hortelã & $23,7 \mathrm{~A}$ & $24,7 \mathrm{~A}$ & & $1693,0 \mathrm{~A}$ & $2408,3 \mathrm{~A}$ \\
Manjericão & $58,3 \mathrm{~A}$ & $64,7 \mathrm{~A}$ & & $2429,4 \mathrm{~A}$ & $3444,1 \mathrm{~A}$ \\
\hline
\end{tabular}

Médias seguidas por pelo menos uma mesma letra nas linhas, não diferem entre si ao nível de $5 \%$ de probabilidade pelo teste $\mathrm{T}$, para cada variável estudada (averages followed by the same capital letter in the lines do not differ statistically between each other; $\mathrm{T}=5 \%$ ).

consorciados com arruda, que tiveram também maior produtividade absoluta total (Tabela 2).

Dentre os insetos presentes na área experimental, os agentes causadores da broca pequena (Neoleocinoides elegantalis) e da broca grande (Spodoptera $s p$. e Trichoplusia $s p$ ) destacaram-se pela maior incidência e danos. Isto corrobora a afirmativa de Paula et al. (2004), que relataram que dentre os insetos-praga que acometem a cultura, os broqueadores destacam-se dentre os mais importantes, por constituírem pragas diretas que atacam a parte de interesse comercial.

O broqueamento dos frutos contribuiu na redução da produção comercial (Tabela 2), com maior comprometimento da produção de tomates nas áreas de monocultivo $(43,64 \%)$ e nas áreas de consórcio com hortelã-pimenta $(46,72 \%)$ e menor nos consórcios com funcho $(24,06 \%)$, que não diferiram significativamente dos consórcios com manjericão $(29,37 \%)$ e arruda $(33,20 \%$; Tabela 2).

As plantas de funcho, devido às maiores altura e área de dossel (Tabelas 1 e 2), funcionaram, provavelmente, como barreira física para o agente causal da broca, enquanto que a hortelã-pimenta, em função das suas características intrínsecas, não atuou como barreira física para o agente causal da broca, e teve pouca possibilidade de sua parte aérea interagir com a parte aérea dos tomateiros. No entanto, ofereceu boa cobertura viva de solo, contribuindo com a redução de plantas infestantes ou daninhas na área de cultivo e manuten- ção da umidade do solo.

Como já discutido, em relação à produção total, o funcho não favoreceu aumento da produção comercial e da produtividade do tomateiro na densidade de plantio adotada (Tabela 2). Isso provavelmente se deveu à similaridade de nicho ecológico e competição. Tendo como referência a produtividade total do tomateiro solteiro, o funcho, o manjericão e a hortelã-pimenta causaram, em média, redução de $64,9 \%, 8,4 \%$ e $1 \%$, respectivamente, na produtividade total do tomateiro, enquanto a arruda causou, em média, aumento de $26 \%$ (Tabela 2).

A arruda e o manjericão favoreceram redução da perda de frutos por broqueamento e, consequentemente, aumento na produtividade comercial: de $9,5 \mathrm{t} \mathrm{ha}^{-1}$, no monocultivo para 17,6 e $12,8 \mathrm{tha}^{-1}$, respectivamente nos consórcios com arruda e com manjericão (Tabela 2). No caso particular da arruda, sugere-se que o nicho ecológico dessa planta seja distinto do nicho do tomateiro, o que favoreceu a redução da competição interespecífica e beneficiou o tomateiro no processo de interação biótica.

Em relação à hortelã-pimenta e ao manjericão, sugere-se que as pressões competitivas líquidas dessas plantas sobre o tomateiro foram insuficientes para reduzirem significativamente a produtividade total do tomateiro.

Os valores de produtividade relativa do tomateiro confirmaram esses dados: maior produtividade relativa no consórcio com arruda $\left(13,6\right.$ t.ha $\left.{ }^{-1}\right)$, seguida pelos consórcios com hortelã-pimenta 
Tabela 2. Frutos broqueados, produção total e comercial de tomates, obtidos em área útil de $6 \mathrm{~m}^{2}$, e produtividade absoluta total e comercial de tomates em cultivo solteiro e consorciado com arruda, funcho, hortelã-pimenta e manjericão (bored fruits, total and commercial yield of tomato, in $6 \mathrm{~m}^{2}$ of area, absolute total and commercial yield of tomato, in single and intercropping tomato and rue, fennel, peppermint and basil). Umbaúba, Embrapa Tabuleiros Costeiros, 2006.

\begin{tabular}{|c|c|c|c|c|c|}
\hline \multirow{2}{*}{ Tratamento } & \multirow{2}{*}{$\begin{array}{c}\text { Frutos broqueados } \\
(\%)\end{array}$} & \multicolumn{2}{|c|}{ Produção da parcela útil (kg) } & \multicolumn{2}{|c|}{ Produtividade absoluta (t ha-1) } \\
\hline & & Comercial & Total & Comercial & Total \\
\hline Tomate solteiro & $43,64 \mathrm{a}$ & $5,68 \mathrm{~b}$ & $12,23 \mathrm{ab}$ & $9,5 \mathrm{~b}$ & $20,4 \mathrm{ab}$ \\
\hline $\mathrm{T}+$ arruda & $33,20 \mathrm{ab}$ & $10,54 \mathrm{a}$ & $16,68 \mathrm{a}$ & $17,6 \mathrm{a}$ & $27,8 \mathrm{a}$ \\
\hline $\mathrm{T}+$ funcho & $24,06 \mathrm{~b}$ & $5,82 \mathrm{~b}$ & $7,88 \mathrm{~b}$ & $9,7 \mathrm{~b}$ & $13,1 \mathrm{~b}$ \\
\hline $\mathrm{T}+$ hortelã & $46,72 \mathrm{a}$ & $6,06 \mathrm{~b}$ & $11,98 \mathrm{ab}$ & $10,1 \mathrm{~b}$ & $20,0 \mathrm{ab}$ \\
\hline $\mathrm{T}+$ manjericão & $29,37 \mathrm{ab}$ & $7,67 \mathrm{~b}$ & $11,08 \mathrm{ab}$ & $12,8 \mathrm{~b}$ & $18,5 \mathrm{~b}$ \\
\hline
\end{tabular}

Médias seguidas pela mesma letra, nas colunas, não diferem entre si ao nível de $5 \%$ pelo teste Tukey (averages followed by the same letter in columns do not differ statistically between each other, using Tukey test, 5\%).

$\left(9,8\right.$ t.ha $\left.^{-1}\right)$ e com manjericão $\left(9,1\right.$ t.ha $\left.{ }^{-1}\right)$, e menor no consórcio com funcho $(6,4$ t.ha' ${ }^{-1}$; Tabela 3$)$. Esses dados não corroboraram a afirmativa de Bomford (2004) de que a produtividade do tomateiro é significativamente maior em cultivo consorciado do que em monocultivo, independente da espécie consorciada.

Os maiores valores de produtividade relativa do tomateiro no consórcio com a arruda podem ser explicados não só por meio do "princípio da produção competitiva", mas também pelo "princípio da produção facilitada".

Segundo Vandermeer (1989) quando a produtividade relativa é maior do que um, o princípio da produção facilitada deve estar operando de alguma forma. A facilitação ocorre, segundo o mesmo autor, quando o efeito de uma espécie no ambiente, causa resposta positiva na outra espécie.

São muitos os mecanismos que podem contribuir para a produção facilitada. Em se tratando de espécies aromáticas que produzem grande número de compostos bioativos, deve-se considerar que um dos mecanismos que pode estar ocorrendo é alelopatia. Isto, no entanto, não chegou a ser investigado neste trabalho.

Gómez-Rodriguez et al. (2003) verificaram que o cravo de defunto (Tagetes erecta), cultivado em consórcio com tomateiro, propiciou proteção ao último, tanto devido a efeitos alelopáticos contra patógenos causadores de doença, quanto por meio da redução da umidade relativa máxima e como barreira física contra a dispersão de esporos fúngicos.
Na maioria dos consórcios um mecanismo comum é a proteção das plantas pela barreira física, devido a alterações no microclima, causadas por um sombreamento diferencial.

Segundo Sobkowicz \& Tendziagolska (2005) e Montezano \& Peil (2006), pode ocorrer aumento da produtividade em cultivos consorciados nos casos em que há aumento da eficiência biológica das associações, o que pode resultar de diferenças no comprimento do ciclo de crescimento e na arquitetura radicular. Assim, a eficiência depende, muitas vezes, da complementaridade de nichos ecológicos, temporal e ou espacial, entre as culturas.

Quando o período de maior demanda pelos recursos ambientais das culturas consorciadas não é coincidente, a competição entre as mesmas pode ser minimizada. Similarmente, quando as diferenças na arquitetura das plantas favorecem a melhor utilização da luz, água e nutrientes disponíveis, ocorre complementaridade espacial (Sobkowicz \& Tendziagolska, 2005).

Dentre as culturas aromáticas componentes dos consórcios estudados, a primeira a ser colhida foi o manjericão, seguido pela arruda, hortelã-pimenta e funcho. Isso se deu em função do ciclo de vida e do órgão da planta de maior interesse comercial.

Verificou-se nas áreas consorciadas com tomateiro tendência à redução na média de produção de hortelã-pimenta e de manjericão e aumento na produção média de arruda e de funcho (Tabela 3 ). A aromática que rendeu maior produção foi o manjericão: $38,32 \mathrm{~kg}$, no cultivo solteiro, e $28,94 \mathrm{~kg}$ no cultivo consorciado, colhidos em parcela útil de $3 \mathrm{~m}^{2}$ cada. Similarmente, a produtividade absoluta do manjericão tendeu a ser menor nas áreas consorciadas: 96,5 t ha $^{-1}$ no consórcio com tomateiro e 127,7 t ha ${ }^{-1}$ no monocultivo.

Quanto à produtividade relativa das aromáticas estudadas, a arruda e o funcho se destacaram, com valores maiores do que um (Tabela 3), o que indicou maior produtividade dessas plantas no cultivo consorciado.

A produtividade relativa da hortelãpimenta e do manjericão foi, em média, menor do que um (Tabela 3). Sugerese que, para estas plantas, a pressão exercida pelo tomateiro foi superior à competição intraespecífica, enquanto para aquelas a pressão intraespecífica foi maior do que a exercida pelo tomateiro.

A partir disso, infere-se que o princípio operante no consórcio com hortelã e manjericão foi o da produção competitiva, enquanto que no consórcio do tomateiro com funcho e arruda ocorreu competição facilitada.

O UET, em todos os casos estudados, teve valor superior a um (Tabela $3)$. Esses resultados indicam que o consorciamento permitiu maior aproveitamento da área de produção do que o monocultivo, sendo, portanto, mais vantajoso.

Segundo Hiebsch \& McCollum (1987), isto indica que o monocultivo requereu mais área do que o consórcio para fornecer a mesma quantidade de produtos. Vandermeer (1989) cita que 
Tabela 3. Produção, produtividade absoluta e relativa, da arruda, funcho, hortelã-pimenta e manjericão, produtividade relativa dos tomateiros, e índice de uso eficiente da terra (UET) (production, absolute and relative yield, of rue, fennel, peppermint and basil, relative yield of tomato, and land efficient ratio).Umbaúba, Embrapa Tabuleiros Costeiros, 2006.

\begin{tabular}{|c|c|c|c|c|c|}
\hline \multirow{3}{*}{ Tratamentos } & \multicolumn{3}{|c|}{ Aromáticas } & \multirow{3}{*}{$\begin{array}{c}\text { Tomateiro } \\
\text { Produtividade relativa } \\
\left(\mathrm{t} \mathrm{ha}^{-1}\right) \\
\end{array}$} & \multirow{3}{*}{ UET } \\
\hline & \multirow{2}{*}{$\begin{array}{c}\text { Produção da parcela } \\
\text { útil (kg) }\end{array}$} & \multicolumn{2}{|c|}{ Produtividade (t ha-1) } & & \\
\hline & & Absoluta & Relativa & & \\
\hline Arruda (A) & 0,33 & 1,09 & - & - & - \\
\hline Arruda (A) & 0,33 & 1,09 & - & - & - \\
\hline Funcho $(\mathrm{F})$ & 0,29 & 0,97 & - & - & - \\
\hline Hortelã $(H)$ & 1,12 & 3,73 & - & - & - \\
\hline Manjericão (M) & 38,32 & 127,7 & - & - & - \\
\hline Tomate $(\mathrm{T})+\mathrm{A}$ & 0,37 & 1,24 & 1,14 & 13,6 & 2,50 \\
\hline $\mathrm{T}+\mathrm{F}$ & 0,48 & 1,60 & 1,65 & 6,4 & 2,29 \\
\hline $\mathrm{T}+\mathrm{H}$ & 0,77 & 2,60 & 0,69 & 9,8 & 1,67 \\
\hline $\mathrm{T}+\mathrm{M}$ & 28,94 & 96,5 & 0,76 & 9,1 & 1,66 \\
\hline
\end{tabular}

UET superior a um indica vantagem de rendimento para o cultivo consorciado, ou seja "sobreprodutividade" e, ainda, a existência de interferências positivas entre os componentes do consórcio. Isto, em geral, se relaciona com a ocorrência de mecanismos de produção "facilitada". Além disso, indica que qualquer interferência interespecífica negativa existente não é tão intensa quanto a que existe nas monoculturas e, portanto, o cultivo consorciado é mais vantajoso (Vandermeer, 1989; Gliessman, 2001; Connoly et al., 2001; Rao, 2002).

A magnitude do índice UET quantifica o aumento na eficiência biológica, definida pelo consórcio (Hiebsch \& McCollum, 1987). UET maior do que 1,5 , como ocorreu nos consórcios do tomateiro com arruda, hortelã-pimenta, funcho e manjericão (Tabela 3), indica, segundo Gliessmam (2001), que há forte evidência de que a interferência negativa das espécies seja mínima nos consórcios, e que as interferências positivas permitam que, pelo menos um dos membros se dê melhor em consórcio do que em cultivo solteiro.

Concluindo, destaca-se que o cultivo consorciado do tomateiro possibilitou maior aproveitamento da área de produção, com a produtividade do tomateiro igual ou superior à obtida no monocultivo, exceto no caso do funcho, na densidade de plantio adotada. Além disso, verificou-se, dentre as aromáticas estudadas, que o manjericão foi a planta que teve maior produção e produtividade absoluta, propiciando colheitas mensais, representando, assim uma interessante fonte extra de rendimentos, sem no entanto causar redução significativa na produtividade do tomateiro. Essa tecnologia pode ser bastante útil para pequenos agricultores familiares que dispõem de área reduzida para plantio e de poucos recursos financeiros, e que podem ter no manjericão uma alternativa na comercialização como erva condimentar.

A arruda permitiu aumento da produtividade do tomateiro; no entanto, é menos atraente como cultura alternativa, em função do ainda reduzido comércio dessa planta como medicinal.

O funcho causou redução na produtividade absoluta total do tomateiro, mas, por outro lado, permitiu menores perdas na produção em função de brocas. Desse modo sugere-se investigar mais profundamente o efeito do consórcio do funcho com o tomateiro em outras densidades e arranjos de plantio.

A hortelã-pimenta não contribuiu em menores perdas na produção de tomate broqueado, mas pode ser utilizada como cultura alternativa, comercializada como erva condimentar e aromática, uma vez que não causou redução significativa na produção total de tomates.

\section{REFERÊNCIAS}

ALTIERI MA; SILVA EN; NICHOLLS CI. 2003. $O$ papel da biodiversidade no manejo de pragas. Ribeirão Preto: Holos. 226p.
AZEVEDO DMP; BELTRÃO NEM; SANTOS JW; LIMA EF; BATISTA FAS; NOBREGA LB; VIEIRADJ; PEREIRA JR. 1998. Efeito de população de plantas no consórcio mamoneira/ sorgo. Oleoginosas e fibrosas 2: 183-192.

BOMFORD MK. 2004. Yield, pest density, and tomato flavor effects of companion planting in garden-scale studies incorporating tomato, basil, and brussels sprout. Morgantown: Davis College of Agriculture, Forestry and Consumer sciences. 121p. Disponível em: http://orgprints.org/6614. Acessado em 10 abr.2008. (Tese doutorado).

BOWIE MH; WRATTEN SD; WHITE AJ. 1995. Agronomy and phenology of "companion plants" of potential for enhancement of insect biological control. New Zealand Journal of Crop and Horticultural Science 23: 423-427.

BURG IC; MAYER PH. 2006. Alternativas ecológicas para prevenção de pragas e doenças. Francisco Beltrão: Grafit Gráfica e Editora, 3 ed rev. e ampl. 153 p.

CONNOLLY J; GOMAHC; RAHIM K. 2001. The information content of indicators in research. Agriculture, Ecosystems and environment 87: 191-207.

EMBRAPA. 1987. Recomendações para o uso de corretivos, matéria orgânica e fertilizantes para hortaliças no Distrito Federal. Brasília: Centro Nacional de Pesquisa de Hortaliças. $50 \mathrm{p}$.

GAMARRA-ROJAS G; PETERSEN P; LIMA MARCELINO (eds). 2003. Desafios da pesquisa em agroecologia no Nordeste. Encontro Nordestino de pesquisa em agroecologia $_{\mathrm{a}} 1^{\circ}$, Recife: IDT/CNIP; ASPTA, $73 \mathrm{p}$.

GLIESSMAN SR. 2001. Agroecologia: processos ecológicos em agricultura sustentável. Porto Alegre: UFRGS. 653p.

GÓMEZ-RODRIGUEZ O; ZAVALETA-MEJIA E; GONZÁLEZ-HERNÁNDEZ VA; LIVERAMUÑOZM; CÁRCENAS-SORIANOE. 2003. Allelopathy and microclimatic modification of marigold on tomato early blight disease development. Field Crops Research 83: 27-34. 
GRANGEIRO LC; NEGREIROS MZ; SANTOS AP; COSTA LM; SILVA ARC; LUCENA RRM. 2008. Crescimento e produtividade de coentro e rabanete em função da época de estabelecimento do consórcio. Ciência e Agrotecnologia 32: 55-60.

HIDDINK GA; TERMORSSHUIZEN AJ; RAAIJMAKERS JM; VAN BRUGGEN AHC. 2005. Effect of mixed and single crops on disease suppressiveness of soils. Phytopathology 95: 1325-1332.

HIEBSCH CK; MCCOLLUM RE. 1987. Area-X time equivalency ratio: a method for evaluating the yield of intercroppings. Agronomy Journal 79: 15-22.

JOLLIFFE PA; WANJAU FM. 1999. Competition and yield in crop mixtures: some properties of productive intercroppings. Journal of Agricultural Science 132: 425-435.

LOOS RA; SILVA DJH; FONTES PCR; PICANÇO MC; GONTIJO LM; SILVA EM; SEMEÃO AA. 2004. Identificação e quantificação dos componentes de perdas de produção do tomateiro. Horticultura Brasileira 22: 1-6.

MAIA JTLS; GUILHERME DO; PAULINO MAO; BARBOSA FS; FERNANDES RC; MAIO MM; VALADARES SV; COSTA CA; MARTINS ER. 2008. Produção de alface e cenoura em cultivo solteiro e consorciado com manjericão e hortelã. Revista Brasileira de Agroecologia 3: 58-64.

MARTINS ER; CASTELLANI DC; CASTRO DM; DIAS JE. 1995. Plantas Medicinais. Viçosa: UFV, Imprensa Universitária. 220 p.

MATSON PA; PARTON WJ; POWER AG; SWIFT MJ. 1997. Agricultural intensification and ecosystem properties. Science 277: 504509.

MELO PCT; VILELA NJ. 2005. Desafios e perspectivas para a cadeia brasileira do tomate para processamento industrial. Horticultura Brasileira 23: 154-157.

MONTEZANO EM; PEIL RMN. 2006. Sistemas de consórcio na produção de hortaliças. Revista Brasileira de Agrociência 12: 129-132.

MORAES AA; VIEIRAMC; HEREDIAZÁRATE NA. 2007. Produção de repolho "Chato de quintal" e da capuchinha "Jewel", solteiros e consorciados, sem e com cama de frango semidecomposta incorporada no solo. Ciência e Agrotecnologia 31: 731-738.

NASCIMENTO E; MOTA JH; VIEIRA MC; HEREDIA ZARATE NA. 2007. Produção de biomassa de Pfaffia glomerata (Spreng.) Pedersen e Plantago major L. em cultivo solteiro e consorciado. Ciência e Agrotecnologia 31: 724-730.

OLIVEIRA FL; RIBAS RGT; JUNQUEIRA RM; PADOVAN MP; GUERRA JGM; ALMEIDA DL; RIBEIRO RLD. 2005. Desempenho do consórcio entre repolho e rabanete com précultivo de crotalária, sob manejo orgânico. Horticultura brasileira 23: 184-188.

PATRA DD; PRASAD A; ANWAR M; SINGH D; CHAND S; RAM B; KATIYAR RS; KUMAR S. 2002. Performance of lemongrass cultivars intercropping with chamomile under sodic soils with different levels of gypsum application. Communications of soil science of plant analyses 33: 1707-1721.

PAULA SV; PICANÇO MC; OLIVEIRA IR; GUSMÃO MR. 2004. Controle de broqueadores de frutos de tomateiro com uso de faixas de culturas circundantes. Bioscience Journal 20: 33-39.

PICANÇO M; FALEIRO FG; PALLINI FILHOA; MATIOLI AL. 1997. Perdas na produtividade do tomateiro em sistemas alternativos de controle fitossanitário. Horticultura Brasileira
15: 88-91.

RAO BRR. 1999. Biomass and essential oil yields of cornmint (Mentha arvensis L. cf. piperascens Malinvaud ex Holmes) planted in different months in semi-arid tropical climate. Industrial crops and products 10: 107-113.

RAO BRR. 2002. Biomass yield, essential oil yield and essential oil composition of rosescented geranium (Pelargonium species) as influenced by row spacings and with cornmint (Mentha arvensis L. cf. piperascens Malinv. ex Holmes). Industrial crops and products 16:133-144.

ROEL AR. 2001. Utilização de plantas com propriedades inseticidas: uma contribuição para o desenvolvimento rural sustentável. Revista Internacional de Desenvolvimento Local 1: 43-50.

SANTOS RHS; GLIESSMAN S; CECON PR. 2002. Crop interactions in broccoli. Biological Agricultural and Horticulture 20: 51-75.

SOBKOWICZ P; TENDZIAGOLSKA E. 2005. Competition and yield in mixture of Oats and wheat. Journal of agronomy and Crop science 191: 377-385.

SOBRAL LF; VIÉGAS PRA; SIQUEIRA OJW; ANJOS JL; BARRETO MCV; GOMES JBV. 2007. Recomendações para o uso de corretivos e fertilizantes no Estado de Sergipe. 1. ed. Aracaju: Embrapa Tabuleiros Costeiros. $251 \mathrm{p}$.

SOUZA JL; RESENDE P. 2003. Manual de horticultura orgânica. Viçosa: Aprenda Fácil. $564 \mathrm{p}$.

TEIXEIRA IR; MOTA JH; SILVA AG. 2005. Consórcio de hortaliças. Semina. Ciências agrárias 26: 507-514.

VANDERMEER J. 1989. The ecology of intercrop. Cambridge: Cambridge University Press. 237 p. 